\title{
Induced Optical Bistability in a Composite of Ellipsoidal Metal/Dielectric Nanoparticles of Dielectric Core
}

\author{
Tolasa Tamasgen ${ }^{1}$, Sisay Shewamare ${ }^{2}$, Getnet Melese ${ }^{3, *}$ \\ ${ }^{1}$ Department of Physics, Jimma University, Ethiopia \\ ${ }^{2}$ Department of Physics, Wolkite University, Ethiopia \\ ${ }^{3}$ Department of Physics, Debre Tabor University, Ethiopia
}

Copyright $\mathrm{C} 2016$ by authors, all rights reserved. Authors agree that this article remains permanently open access under the terms of the Creative Commons Attribution License 4.0 International License

\begin{abstract}
In this paper, we studied the enhancement factor of local field for ellipsoidal metal composite, and optical induced bistability of metal/dielectric composites with in linear host matrixes. Using the calculated enhancement factor of local field and the cubic equation of the optical induced bistability of the composite material, the parameters of the bistability domain are calculated. The analytical and numerical results show that the enhancement factor of local field is extremely enhanced and the optical induced bistability increases its domain. It is shown that the local field in metal ellipsoidal particles with dielectric core in an external varying electric field has two maxima at two different frequencies. The second maximum becomes more important with increment in the metal fraction. At high metal fraction, the two bistability domains merge and form one entire bistability domain.
\end{abstract}

Keywords Dielectric Function, Optical Bistability, Enhancement Factor, Depolarization Factor

\section{Introduction}

Recent advances in nanotechnology have allowed the development of robust, and highly sensitive and selective detection methods that are expected to address some deficiencies of conventional detection technologies. Within this context gold $(\mathrm{Au})$, and silver $(\mathrm{Ag})$, nanoparticles have emerged as a powerful tool in sensing and imaging optical applications due to their surprising optical properties. Although silver exhibits many advantages over gold, such as higher extinction coefficients, sharper extinction bands, higher ratio of scattering to extinction, and extremely high field enhancements. It has been employed in the development of sensors, based on surface enhanced spectroscopes. The reason for this is the lower chemical stability of silver nanoparticles when compared to gold. As a consequence, silver nanoparticles are rapidly gaining in popularity and several research groups have began to explore alternative strategies for the development of optical sensors and imaging labels based on the extraordinary optical properties of these metal nanoparticles [1].

Interaction of light with nanocomposites reveals novel optical phenomena indicating unrivalled optical properties of these materials. The linear and nonlinear optical response of metal nanoparticles is specified by oscillations of the surface electrons in the Coulomb potential formed by the positively charged ionic core. During the last century optical properties of nanoparticles have extensively been studied and metal-dielectric nanocomposites have found various applications in different fields of science and technology. Since the optical properties of metal nanoparticles are governed by surface plasmon resonance (SPR), and they are strongly dependent on the nanoparticles' size, shape, concentration and spatial distribution as well as on the properties of the surrounding matrix [2].

The physical requirements for optical bistability (OB) are an intensity-dependence, refractive index and an optical feedback mechanism. A system is said to be optically bistable if it can exhibit two steady output states for the same input intensity over some range of input values. The switching up and down operations typical in a hysteresis cycle originates from the rise of instability. A physical state is said to be unstable when, after displaying the system a little from this steady point, the system does not return to it and goes further from it. In other words, for an unstable state, even the slightest perturbation removes the system from it. The searches for instabilities turn out to be crucial in the study of optical bistability (OB) phenomena not only from the theoretical viewpoint but also for the possibilities for practical and technological applications. Since its first discovery in late 1970's, optical bistability has been found existing in many different optical systems. One of the simplest examples of bistable systems is a Fabry-Perot resonator with the cavity filled with a medium that presents saturable absorption or nonlinear dispersion [3 -5].

These optical properties of silver nanoparticles have been recently studied extensively and metal dielectric composite have found various applications in different fields of science 
and technology site. The original study of the effective optical properties of composite materials were to the analysis of linear media and by considering the contribution of the local field of the particle it is extended to study of the materials with nonlinear part. This is strongly dependent on the nanoparticles size, shape, volume fraction, spatial distribution and the properties of the surrounding matrix. Control over these parameters enables such metal/dielectric nanocomposite to become promising media for development of precious/novel nonlinear materials in nano devices and optical elements [6-8].

The two main purposes of metal/dielectric composite are: allowing light to enter deeply in the metals, and achieving metal localization which in turn leads to an enhancement of nonlinear response. Another interesting property of metal nanoparticle is the presence of extinction bands in the visible or infrared that results from the so called plasmon resonances. These resonances do not exist in bulk metals and can be explained to be a consequence of the confinement of free electrons in a space smaller than one-wavelength of light which can be controlled by changing the shape of the nanoparticle and its orientation with respect to the electric field. Furthermore, very small concentration of the nonlinear part contributions can be treated as real and as small perturbations (low field approximation can be assumed) leading which can be assumed to be resulting in linear behavior [9-12].

Nonlinear optical properties are very important in many different applications in areas of science and technology, such as nano nonlinear microscopy [13], ultra fast laser system [14], wavelength conversion [15], optical routing and switching based on optically induced bistability both on network [16, 17], and on chips [18]. It is known that metals have fast and strong nonlinear response [19], and can be good element for nonlinear optical applications when they are composed with dielectrics [20,21].

It has been commonly acknowledged that all-optical devices at the micrometer and nanometer scales is a promising way towards realization of next-generation ultrafast communication and signal processing systems beyond today's microelectronics devices, which have gradually encountered limitation in bandwidth and speed. Optical switching is an essential component in the all-optical network. A feasible approach to all-optical switching is based on optical bistability, an important subject in nonlinear optics [22]. Optical bistability offers many intriguing applications, such as optical memory and optical transistor [23 - 27], and among others. In recent years there has been a great interest in exploring and realizing optical bistability in nonlinear nanophotonic systems.

\section{Analytically and Numerically Study of Enhancement Factor in the Small Ellipsoidal Metal/Dielectric with Composite of Dielectric Core}

The optical response for a collection of free electrons can be obtained from the Lorentz harmonic oscillator model by simply 'climbing the springs' that is by setting the spring constant $\mathrm{k}$ is equal to zero. Therefore, for $\omega$ is incident frequency and dielectric function for free electron is given by;

$$
\varepsilon(\omega)=\varepsilon_{\infty}-\frac{\omega_{p}^{2}}{\omega^{2}+i v \omega}
$$

where $\varepsilon(\omega)$ is dielectric function as a function angular velocity, $\omega_{p}$ is plasma frequency and $\varepsilon_{\infty}$ the high frequency dielectric constant.

Let an electromagnetic wave impinge on a metal particle in the form of a rotational ellipsoid embedded in a dielectric host matrix. The dielectric function of the particle is assumed to depend on the frequency $\omega$ and the local electric field can be presented in the form

$$
\epsilon(\omega, E)=\epsilon(\omega)+\chi(\omega)|E|^{2}
$$

where, $\chi(\omega)$ is the complex Kerr coefficient. The linear parts of dielectric function is

$$
\epsilon(\omega)=\epsilon^{\prime}(\omega)+i \epsilon^{\prime \prime}(\omega)
$$

where $\epsilon^{\prime}(\omega)$ is the real part of dielectric function and $\epsilon^{\prime \prime}(\omega)$ is the imaginary part of dielectric function.

From eqn (3) let us have both real and imaginary parts by its conjugate;

$$
\begin{gathered}
\epsilon^{\prime}(\omega)=\epsilon_{\infty}^{\prime}-\frac{\omega_{p}^{2}}{\left(\omega^{2}+v^{2}\right)} \\
\epsilon^{\prime \prime}(\omega)=\epsilon_{\infty}^{\prime \prime}+\frac{\omega_{p}^{2} v}{\omega\left(\omega^{2}+v^{2}\right)}
\end{gathered}
$$

\section{The Enhancement of Local Field in the Small Ellipsoidal Metal/Dielectric Particles}

The value of the microscopic field at the position of the atom is called the local field. The local field $E_{L F}(r)$ is different from the applied electric field $E_{0}$ and from the macroscopic electric field $E$.

From the size dependence of the surface plasmon (SP) frequency, it is quite obvious that metal nanoparticles with non-spherical shape will show several SP resonances in their spectra. For instance, ellipsoidal particles with axes $\mathrm{a}=\mathrm{b}=\mathrm{c}$ spherical posses three SP modes corresponding to polarizabilities along the principal axes. Moreover, an increase in the axis length leads to the minimization of the depolarization factor L. For a spherical [5] particle $L_{a}=$ $L_{b}=L_{c}=1 / 3$. Thus, if the propagation direction and polarization of the electromagnetic wave do not coincide with the axes of the ellipsoid, the extinction spectra can demonstrate three separate SP bands corresponding to the oscillations of the free electrons along these axes.

However, if the incident light is polarized parallel to one of the axes, only one single SP band exist corresponding to the appropriate axis. In the case where two SP resonances are observed the band lying at higher wavelengths is referred to 
as the long axis, while the small axis demonstrates resonance at shorter wavelengths compared to the single resonance of a nanosphere of the same volume. The spectral separation of the two surface plasmon bands of the ellipsoidal nanoparticle strongly depends on its aspect ratio which is defined as the ratio of the long to the short axes. At the same time, it is clearly seen that for prolate and oblate spheroids having the same aspect ratio, the positions of SP resonances are different. Namely, the spectral separation between SP bands is higher for the nanoparticles having a zeppelin-like shape [5].

\section{Enhancement Factor of Local Field for Metal/Dielectric Composite}

Consider a composite system that consists of an ellipsoidal shaped dielectric core of dielectric constant $\varepsilon_{d}$, a metal/semiconductor particle with dielectric function $\varepsilon_{m}$ embedded in host matrix having a dielectric function $\varepsilon_{h}$ is as shown Fig.1.1. Using this we will derive enhancement factor $A$ for ellipsoid particle by using this spherical coordinates as reference. Now, we have to use the depolarization factor to shift spherical into ellipsoid shape particle [5].

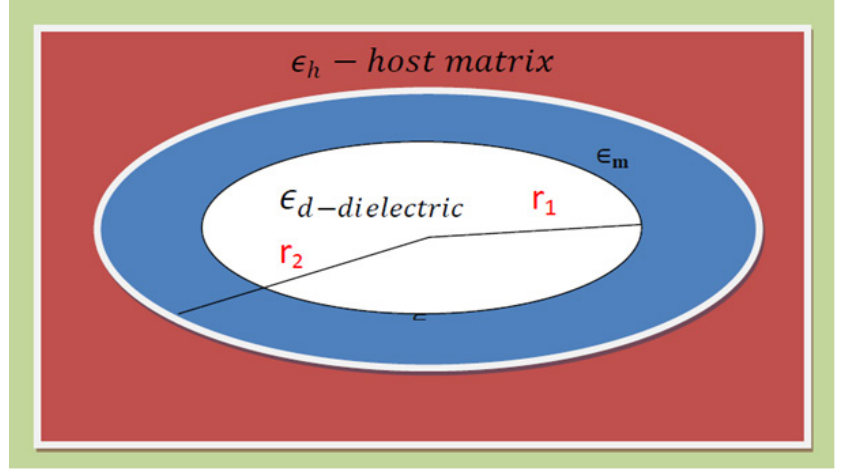

Figure 1. The construction of ellipsoidal by the combination of dielectric $\varepsilon_{d}$ in core, metallic dielectric $\varepsilon_{m}$ outside coverage of dielectric and finally host matrix $\varepsilon_{h}$ external coverage of dielectric and metallic.

The distribution of the electric potential in the system is described by the solutions of the Laplace equations of the dielectric core, metal and the host matrix, respectively.

$$
\begin{gathered}
\Phi_{1}=-E_{h} A r \cos \theta, r \leq r_{1} \\
\Phi_{2}=-E_{h}\left(B r-\frac{C}{r^{2}}\right) \cos \theta, r_{1} \leq r \geq r_{2} \\
\phi_{3}=-E_{h}\left(r-\frac{D}{r^{2}}\right) \cos \theta, r>r_{2}
\end{gathered}
$$

Here $\Phi_{1}, \Phi_{2}$, and $\phi_{3}$ are potentials in the dielectric core, metal, and the host matrix, respectively, $\mathrm{E} \mathrm{h}$ is the applied field, $r$ and $\theta$ are the usaual spherical coordinates of the observation point (the z-axis is chosen along the vector $\left.E_{h}\right), r_{1}, r_{2}$ are the radii of the dielectric core and the metal shell, respectively. $A, B, C, D$ are coefficients that can be determined by boundary conditions.

Applying the continuity conditions of the potential at the boundaries of dielectric core and metal/dielectric cover we get:

$$
A=B-\frac{C}{r_{1}^{3}}
$$

and

$$
B-\frac{C}{r_{2}^{3}}=1-\frac{D}{r_{2}^{3}}
$$

Again applying the continuity conditions of the displacement vector at the boundaries of dielectric core and metal/dielectric cover we have:

$$
\varepsilon_{d} A=\varepsilon_{m}\left(B+\frac{2 C}{r_{1}^{3}}\right)
$$

By introducing the depolarization factor $\mathrm{L}$ in the equation (11) of spherical equation setting that have ellipsoidal shape and comparing with spherical shape of metal/dielectric particles,

$$
L A=\frac{\varepsilon_{m}}{\varepsilon_{d}}[L(B)+(1-L)] \frac{C}{r_{1}^{3}}
$$

Applying the boundary condition for the displacement vector at the metal/dielectric and host matrix interface, we get:

$$
\epsilon_{m}\left(B+\frac{2 C}{r_{2}^{3}}\right)=\epsilon_{h}\left(1+\frac{2 D}{r_{2}^{3}}\right)
$$

And introducing depolarization factor in equation (13),

$$
\epsilon_{m} B+\epsilon_{m}(1-L) \frac{C}{r_{2}^{3}}=L \epsilon_{h}+\epsilon_{h}(1-L) \frac{D}{r_{2}^{3}}
$$

If we plug equation (9) into (14), we get:

$$
\mathrm{B}=\frac{C}{r_{1}^{3}} \frac{\left(L \epsilon_{d}+(1-L) \epsilon_{m}\right)}{L\left(\epsilon_{d}-\epsilon_{m}\right)}
$$

Again, plug equation (15) into (12), here, new definition is introduced what we called volume fraction as:

$$
\frac{r_{1}}{r_{2}}=1-P
$$

Again put (15) into (10) we have:

$$
\frac{C}{r_{1}^{3}}\left[\frac{L \epsilon_{d}+\epsilon_{m(1-L)-(1-P)\left(L \epsilon_{d}+L \epsilon_{m}\right)}}{L\left(\epsilon_{d}+\epsilon_{m}\right)}\right]=1-\frac{D}{r_{2}^{3}}
$$

and equation (14) becomes

$$
\begin{gathered}
\frac{C}{r_{1}^{3}}\left[\frac{\epsilon_{m}^{2} L(1-L)+L^{2} \epsilon_{d} \epsilon_{m}+L\left(\epsilon_{d}+\epsilon_{m}\right) \epsilon_{m(1-L)-(1-P)}}{L\left(\epsilon_{d}+\epsilon_{m}\right)}\right]=L \epsilon_{h}+ \\
\epsilon_{h}(1-L) \frac{D}{r_{2}^{3}}
\end{gathered}
$$

Taking the ratio of equation (17) and equation (18), we get:

$$
\frac{\epsilon_{m}+P L\left(\epsilon_{d}-\epsilon_{m}\right)}{L \varepsilon_{\mathrm{m}} \varepsilon_{\mathrm{d}}(1-\mathrm{P})+\varepsilon_{\mathrm{m}} \mathrm{LP}\left(\varepsilon_{\mathrm{d}}-\varepsilon_{\mathrm{m}}\right)+\mathrm{P} \varepsilon_{\mathrm{m}}^{2}}=\frac{1-\frac{D}{r_{2}{ }^{3}}}{L \epsilon_{h}+\epsilon_{h}(1-L) \frac{D}{r_{2}^{3}}}
$$

Let

$$
\begin{gathered}
a=\varepsilon_{\mathrm{m}} \varepsilon_{\mathrm{d}}-\mathrm{P} \varepsilon_{\mathrm{m}}\left(\varepsilon_{\mathrm{d}}-\varepsilon_{\mathrm{m}}\right)+\mathrm{LP} \varepsilon_{\mathrm{m}}\left(\varepsilon_{\mathrm{d}}-\varepsilon_{\mathrm{m}}\right) \\
b=\varepsilon_{m}+p L\left(\boldsymbol{\epsilon}_{\boldsymbol{d}}-\boldsymbol{\epsilon}_{\mathrm{m}}\right) \\
\frac{b}{a}=\frac{1-\frac{D}{r_{2}{ }^{3}}}{L \epsilon_{h}+\epsilon_{h}(1-L) \frac{D}{r_{2}^{3}}}
\end{gathered}
$$


Thus, the coefficients in the solution of Laplace's equation will be:

$$
\begin{gathered}
A=\frac{\varepsilon_{h} \varepsilon_{m}}{L a+b \varepsilon_{h}(1-L)} \\
B=\frac{C}{r_{1}^{3}} \frac{(1-L) \varepsilon_{m}+L \varepsilon_{d}}{\varepsilon_{d}-\varepsilon_{m}} \\
\mathrm{C}=\frac{\left(\varepsilon_{d}-\varepsilon_{m}\right) \varepsilon_{h}}{L a+b \varepsilon_{h}(1-L)} r_{2}^{3} \\
\mathrm{D}=\frac{\left(a-b \varepsilon_{h}\right)}{L a+b \varepsilon_{h}(1-L)} r_{2}^{3}
\end{gathered}
$$

Plugging the values of $a$ and $b$, the constants can be written as:

$$
\begin{array}{cc}
\varepsilon_{h} \varepsilon_{m} & A= \\
\varepsilon_{\mathrm{m}}^{2}(\mathrm{LP}(1-\mathrm{L}))+\varepsilon_{\mathrm{m}}\left[\varepsilon_{h}(1-L)-\varepsilon_{h} L P(1-L)+L \varepsilon_{\mathrm{d}}(1-\mathrm{P})+\mathrm{L}^{2} \mathrm{P} \varepsilon_{\mathrm{d}}\right]+\varepsilon_{\mathrm{h}} \varepsilon_{\mathrm{d}} \mathrm{LP}(1-\mathrm{L})
\end{array}
$$

Let's denote

$$
\begin{gathered}
a_{1}=\mathrm{LP}(1-\mathrm{L}) \\
a_{2}=\varepsilon_{h}(1-L)-\varepsilon_{h} L P(1-L)+L \varepsilon_{\mathrm{d}}(1-\mathrm{P})+\mathrm{L}^{2} \mathrm{P} \varepsilon_{\mathrm{d}} \\
a_{3}=\varepsilon_{\mathrm{h}} \varepsilon_{\mathrm{d}} \mathrm{LP}(1-\mathrm{L})
\end{gathered}
$$

Therefore, equation (25) becomes

$$
A=\frac{\varepsilon_{h} \varepsilon_{m}}{a_{1} \varepsilon_{\mathrm{m}}^{2}+a_{2} \varepsilon_{\mathrm{m}}+a_{3}}
$$

Recall that the dielectric function of the metal/dielectric inclusion is given by

$$
\varepsilon_{m}=\varepsilon_{m}^{\prime}+i \varepsilon_{m}^{\prime \prime}
$$

By substituting this metallic dielectric into enhancement factor value of $\mathrm{A}$, we get the following equation:

$$
|A|^{2}=\frac{\left|\varepsilon_{h}\right|^{2}\left(\varepsilon_{m}^{\prime}{ }^{2}-\varepsilon_{m}^{\prime \prime}{ }^{2}\right)}{\left(a_{1}\left(\varepsilon_{m}^{\prime}{ }^{2}-\varepsilon_{m}^{\prime \prime}{ }^{2}\right)+a_{2} \varepsilon_{m}^{\prime}+a_{3}\right)^{2}+\left(2 a_{1} \varepsilon_{m}^{\prime} \varepsilon_{m}^{\prime \prime}+a_{2} \varepsilon_{m}^{\prime \prime}\right)^{2}}
$$

This equation is called enhancement factor of unknown coefficient from our initial equation, where the metallic dielectric function from Lorentz classical model is given by the equation:

$$
\begin{gathered}
\varepsilon_{m}^{\prime}=\varepsilon_{\infty}^{\prime}-\frac{1}{z^{2}+\gamma^{2}} \\
\varepsilon_{m}^{\prime \prime}=\varepsilon_{\infty}^{\prime \prime}+\frac{\gamma}{z\left(z^{2}+\gamma^{2}\right)}
\end{gathered}
$$

where, $\varepsilon_{\infty}^{\prime}$ and $\varepsilon^{\prime \prime}{ }_{\infty}$ are constants that can be depend on the type of metal and $\mathrm{z}$ is a dimensionless frequency, where

$$
\left(z=\frac{\omega}{\omega_{p}} \text { and } \gamma=\frac{v}{\omega_{p}}\right) .
$$

\section{Results and Discussions}

\subsection{Enhancement Factor of Local Field for Ellipsoidal Metal/Dielectric Composite in 2D for Depolarization Factor L}

Figure 2 shows that with the change of $\mathrm{L}$ from lower value to high value, namely, with the transition from dielectric property to metallic property, the level values of the enhancement factor of the local field increases. Compared with the case of no dielectric layer, the metal-like dielectric layer makes the threshold values increase; while the dielectric like layer makes the level values decrease. Therefore, the enhancement factor for metallic dielectric function increases, while dielectric function make enhancement factor to decrease.

As we can see from the graph, the maximum enhancement occurs near $\boldsymbol{z}=\mathbf{0 . 3 3}$ and $\boldsymbol{z}=\mathbf{0 . 3 7}$ with peak values 8000 and 7000 respectively.

\subsection{Enhancement Factor of local Field for Ellipsoidal Metal/Dielectric Composite for Oblate and Prolate}

The oblate shaped object have the equatorial diameter greater than the polar diameter, whereas, prolate having the polar diameter greater than the equatorial diameter. Using geometrical factor $\mathrm{L}$ for oblate $\mathrm{L} 1=0.3$ and prolate $\mathrm{L} 2=$ 0.44 , as we can to see from figure 3 no effective change is observed, except when the number value of $\mathrm{L}$ is increased and the enhancement increases from the transition of dielectric to metallic. This means that the enhancement factor increases for large depolarization factor; therefore the maximum enhancement factor is observed for prolate geometry and its peak value is near $z=0.33$ and $z=0.37$. For oblate orientation, the maximum enhancement is observed near $z=0.32$ and $z=0.38$

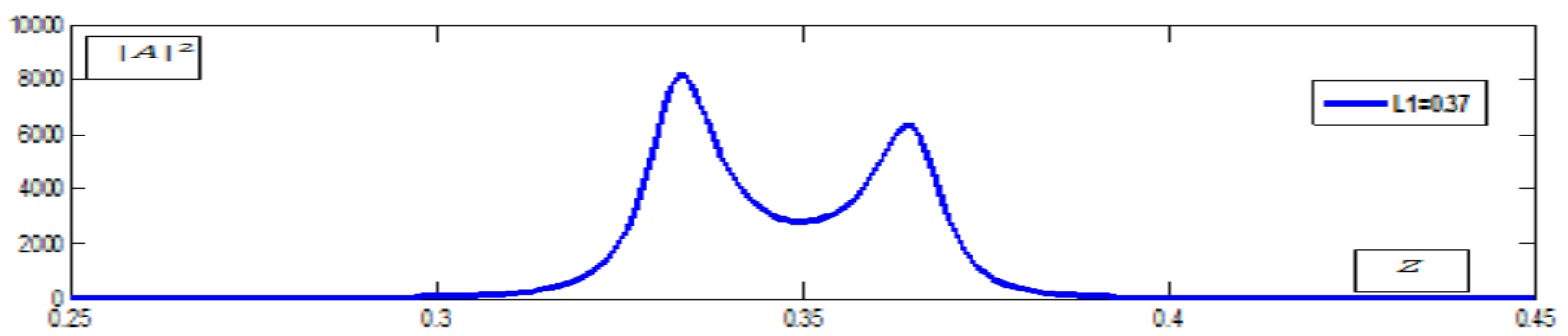

Figure 2. 2D plot of the enhancement factor $|\mathrm{A}|^{2}$ of a silver particle versus $\mathrm{z}, \mathrm{L}=0.37, \omega_{p}=1.46 \times 10^{16} \mathrm{rad} / \mathrm{sec}, \mathrm{v}=1.68 \times 10^{14} \mathrm{rad} / \mathrm{sec}$. 


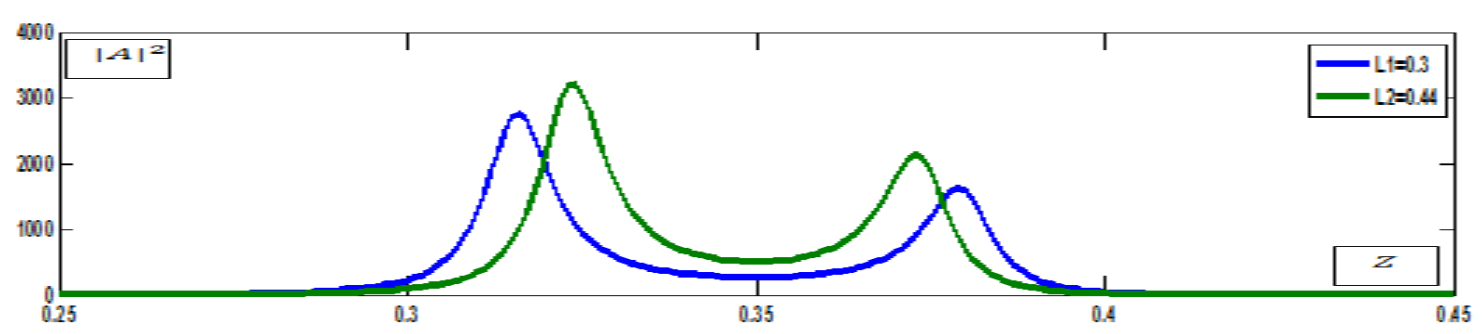

Figure 3. Enhancement factor for the oblate and prolate shape, when $\mathrm{L} 1=0.3$ and $\mathrm{L} 2=0.44$ and other quantities are the same to figure 1

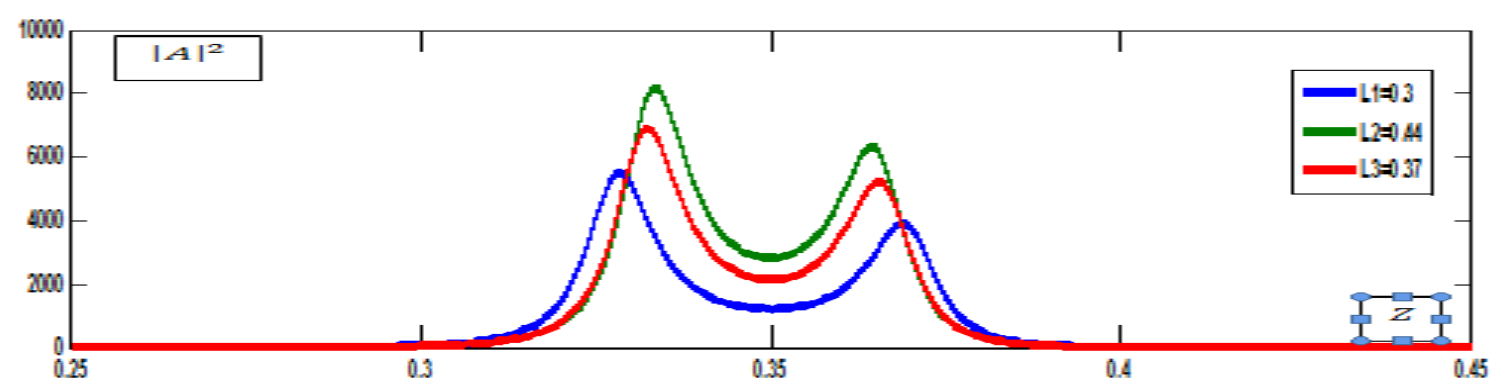

Figure 4. Enhancement factor for the oblate, pure Sphere and prolate shape respectively, when $\mathrm{L} 1=0.3, \mathrm{~L} 2=0.37$ and $\mathrm{L} 3=0.44$ and other quantities are the same to figure 4.

\subsection{Enhancement Factor of Local Field for Ellipsoidal Metal/Dielectric Composite for Sphere, Oblate and Prolate Shape}

Fig. 4 shows the enhancement factor, $|A|^{2}$ of composites of small ellipsoidal metal/dielectric separated by dielectric layer versus the resonance frequency $z$ for three different depolarization factor values; $L 1=0.3, L 2=0.37$ and $L 3=0.44$. We observe that the depolarization factor plays an important role in enhancement factor. It is shown that with the change of $L$ for oblate, prolate and sphere, namely, with the transition of dielectric property to metallic property, the values of the enhancement factor of the local field stay the same for the three shapes.

\subsection{Analytical Description of Induce Optical Bistability for Ellipsoidal Metal/Dielectric Composite Core}

As input intensity is increased, the field inside the cavity also increases, lowering the absorption that the field experiences and thus increasing the field intensity. If the intensity of the incident field is subsequently lowered, the field inside the cavity tends to remain large because the absorption of the material system has already been reduced. Note that over some range of input intensities more than one output intensity is possible. This is called induced optical bistability which means that some nonlinear optical systems can produce two different output intensities for a given input intensities or, in particular, the given value of an external electric field may produce several values for the local field and the polarization. We consider the local field in metal ellipsoidal particles while accounting for the nonlinear part of $\varepsilon(\omega, E)$ and it is used in the presence of small field $\chi|E|^{2}$. From equation (21) we have:

$$
A=\frac{\varepsilon_{h} \varepsilon_{m}}{L a+b \varepsilon_{h}(1-L)}
$$

where $a=\varepsilon_{\mathrm{m}} \varepsilon_{\mathrm{d}}-\mathrm{P} \varepsilon_{\mathrm{m}}\left(\varepsilon_{\mathrm{d}}-\varepsilon_{\mathrm{m}}\right)+\mathrm{LP} \varepsilon_{\mathrm{m}}\left(\varepsilon_{\mathrm{d}}-\varepsilon_{\mathrm{m}}\right)$

$\varepsilon_{\mathrm{d}}=\varepsilon_{\mathrm{do}}+\chi|E|^{2}$ and $\varepsilon_{\mathrm{do}}$ is the real part of dielectric function

After multiplying the above equation we have to separate it in to its real and imaginary as

$$
a=a_{1}+X a_{3}+i\left(a_{2}+X a_{4}\right)
$$

where

$$
\begin{gathered}
a_{1}=\varepsilon_{d o} \varepsilon_{m}^{\prime}(1-P+L P)+\left(\varepsilon_{d}^{\prime 2}-\varepsilon_{m}^{\prime \prime 2}(P-L P)\right) \\
a_{2}=\varepsilon_{d o} \varepsilon_{m}^{\prime \prime}(1-P+L P)+2 \varepsilon_{m}^{\prime} \varepsilon_{m}^{\prime \prime}(P-L P) \\
a_{3}=\varepsilon_{m}^{\prime}(1-P+L P) \\
a_{4}=|E|^{2} \varepsilon_{m}^{\prime \prime}(1-P+L P) \text { and } \\
\mathrm{X}=\chi|E|^{2}
\end{gathered}
$$

And from value of $b$

$$
b=\varepsilon_{m}+L P\left(\varepsilon_{d}-\varepsilon_{m}\right)
$$

By substituting dielectric and metallic function and multiplying, we get

$$
b=\left(a_{5}+X a_{7}\right)+i a_{6}
$$

where,

$$
\begin{gathered}
a_{5}=\varepsilon_{m}^{\prime}(1-L P)+\varepsilon_{d o} L P \\
a_{6}=\varepsilon_{m}^{\prime \prime}(1-L P) \\
a_{7}=L P
\end{gathered}
$$

Plug these values in equation (29) and taking its modulus, we get:

$$
|A|^{2}=\frac{\varepsilon_{h}^{2}\left(\varepsilon_{m}^{2}+\varepsilon^{\prime \prime}{ }_{m}^{2}\right)}{\left(a_{8}+X a_{9}\right)^{2}+i\left(a_{10}+a_{11}\right)^{2}}
$$


where

$$
\begin{gathered}
a_{8}=L a_{1}+(1-L) \varepsilon_{h} a_{5} \\
a_{9}=L a_{3}+\varepsilon_{h}(1-L) a_{7} \\
a_{10}=L a_{2}+a_{6}(1-L) \varepsilon_{h}, \text { and } \\
a_{11}=L a_{4}
\end{gathered}
$$

The combination of all the above equation led us to find the particle at the particular point, starting from

$$
E_{L F}=A\left|E_{h}\right|
$$

Squaring and multiplying both sides of the equation (33) by $\chi$, we get:

$$
\chi\left|E_{L F}\right|^{2}=|A|^{2} \chi\left|E_{h}\right|^{2}
$$

Letting $X=\chi\left|E_{L F}\right|^{2}$, and $Y=\chi\left|E_{h}\right|^{2}$ we obtain the following cubic equation for $X$

$$
Y=\frac{a X^{3}+b X^{2}+c X}{\varepsilon_{h}^{2}\left(\varepsilon_{m}^{2}+\varepsilon^{\prime \prime}{ }_{m}^{2}\right)}
$$

Where

$$
\begin{gathered}
a=a_{9}^{2}+a_{11}^{2} \\
b=2\left(a_{8} a_{9}+a_{10} a_{11}\right) \\
c=a_{8}^{2}+a_{10}^{2}
\end{gathered}
$$

Using equation (35), we draw the optical bistability curve. From figure 5., we understand that when we supply intensity from an external source we get two stable output intensities. We call this stability as bistability that is a real numbers, and there is one imaginary part which is absorbed. Further, we are interested only in the real and positive roots of the above cubic equation. If this equation has one real positive root, then the local field in the inclusion is a single-valued function of the applied field. If equation (35) has three positive roots, then the local field is not a single-valued function of the applied field, and the system becomes unstable. This situation is called the induced optical bistability (IOB). IOB is usually illustrated in the $\mathrm{Y}-\mathrm{X}$ plane and connected with S-like curves showing that three different values of the local field correspond to one value of the applied field.

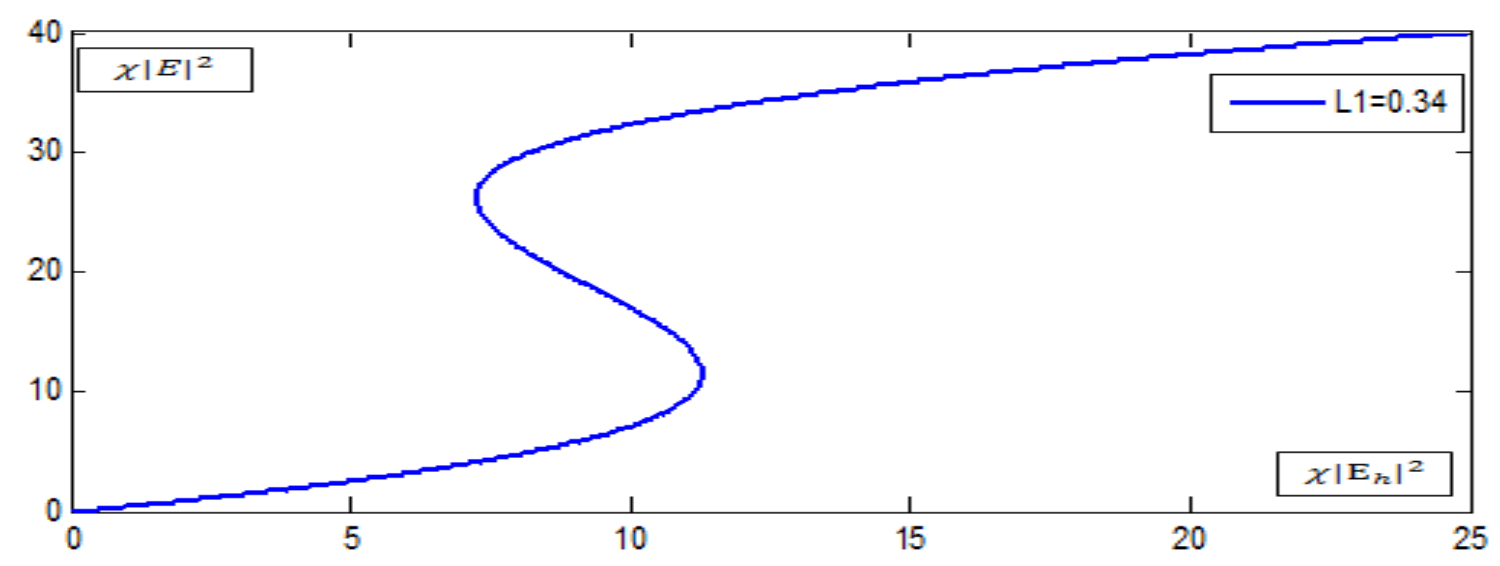

Figure 5. Induced optical bistability in composites of small ellipsoidal metal/dielectric in a linear host matrix for $\mathrm{L} 1=0.37, \varepsilon_{d o}=0.6, \varepsilon_{\infty}=4.5$, where the rest quantities are the same as figure 2 above and this figure is drawn for the local field $\chi\left|E_{L F}\right|^{2}$ as a function of the applied field $\chi\left|E_{h}\right|^{2}$ at $\mathrm{z}=0$.

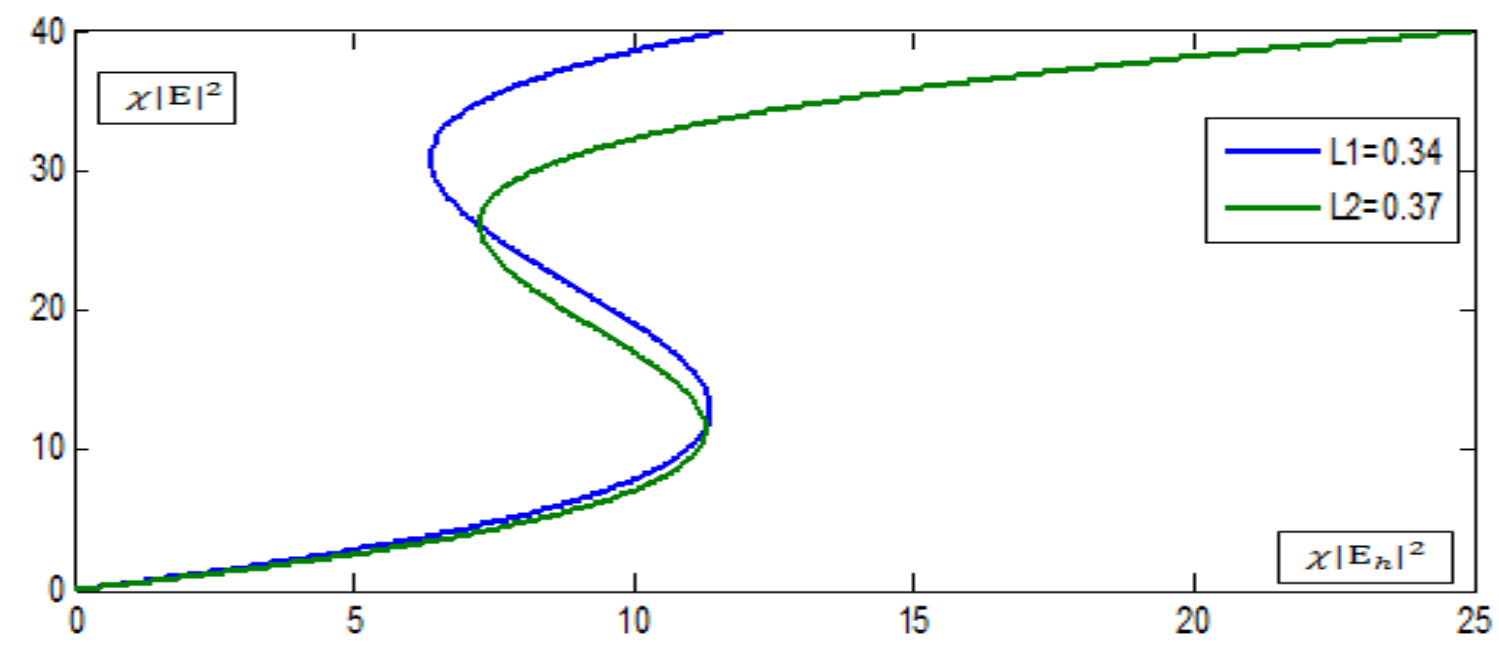

Figure 6. Induced optical bistability in composites of small ellipsoidal metal/dielectric in a linear host matrix for $\mathrm{L}_{1}=0.34, \mathrm{~L} 2=0.37, \varepsilon_{d o}=0.6, \varepsilon_{\infty}=4.5$, where the rest quantities are the same as figure 2 above and this figure is drawn for the local field $\chi\left|E_{L F}\right|^{2}$ as a function of the applied field $\chi\left|E_{h}\right|^{2}$ at $\mathrm{z}=0.2$ 
To obtain the general picture of the connection between the applied field, local field, and the frequency ( $\mathrm{z}$ is constant), we decided to present a $2 \mathrm{D}$ graph involving these quantities. The 2D graph depicted in Fig. 7 is obtained with the help of equation (35) and shows the most interesting region of these parameters (bistability), when three different values of the local field correspond to one value of the applied field.

While analyzing the bistability phenomena in the system, it is more convenient to consider the dependence of a local field on the applied field.

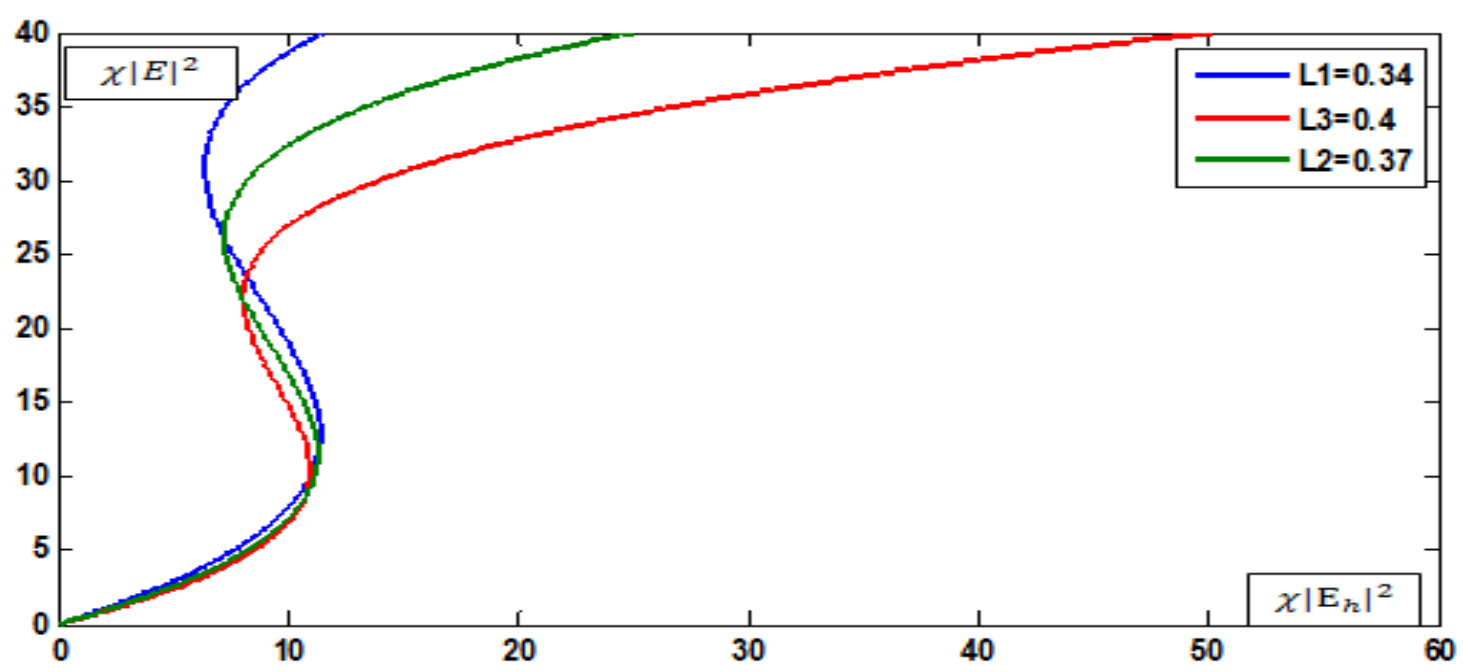

Figure 7. Induced optical bistability in composites of small ellipsoidal metal/dielectric in a linear host matrix for $\mathrm{L}_{1}=0.34, \mathrm{~L} 2=0.37, \mathrm{~L}_{2}=0.40$, $\varepsilon_{d o}=0.6, \varepsilon_{\infty}=4.5$, where the rest quantities are the same as figure 5.1 above and this figure is drawn for the local field $\chi\left|E_{L F}\right|^{2}$ as a function of the applied field $\chi\left|E_{h}\right|^{2}$ at $\mathrm{z}=0.2$

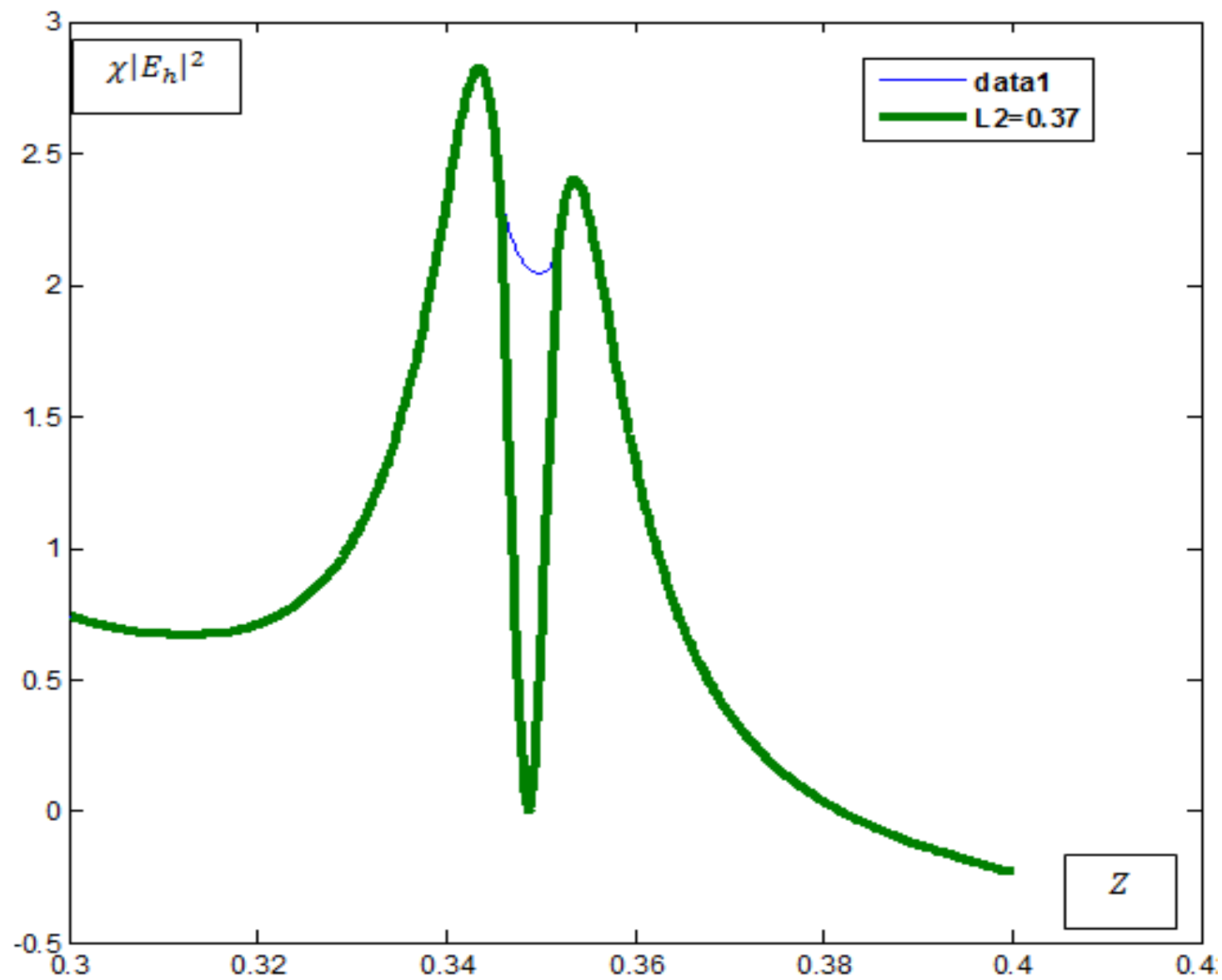

Figure 8. The ellipsoidal silver nanparticle with the parameters $\mathrm{L}=0.37 \varepsilon_{h}=2.25, P=0.99, \gamma=1.15 \times 10^{-2}, \varepsilon_{\infty}=4.5$. The induced optical bistability in the plane $\left(z, \chi\left|E_{h}\right|^{2}\right)$ 


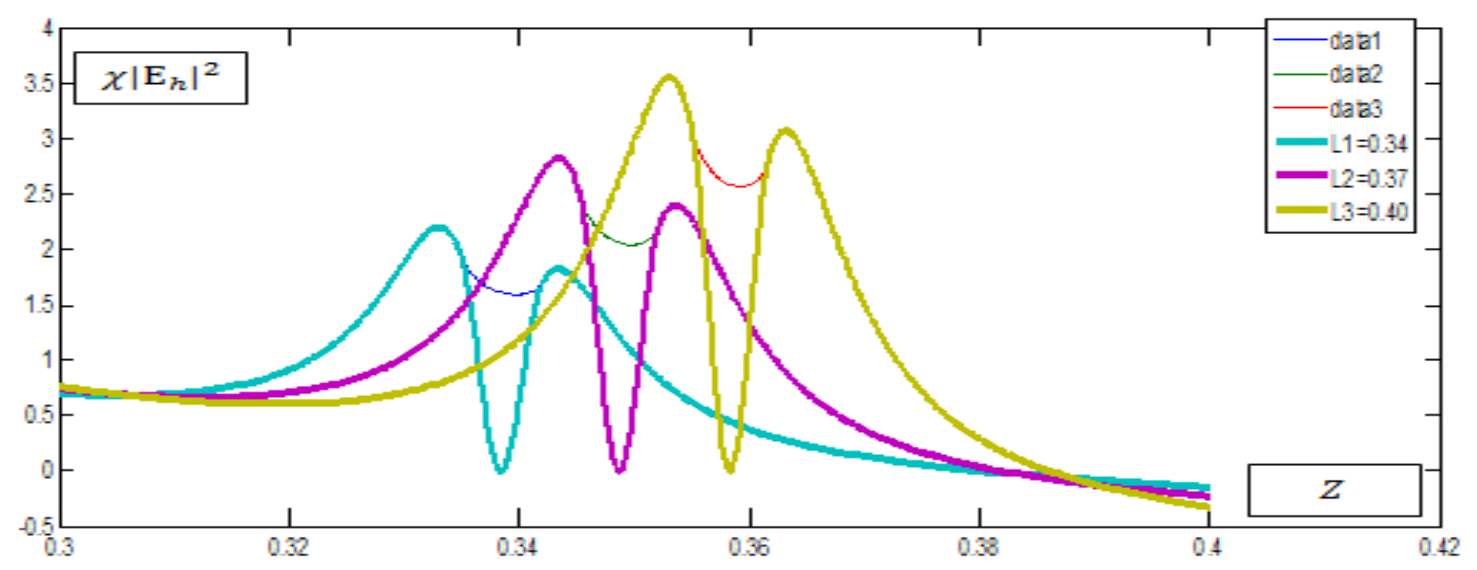

Figure 9. The ellipsoidal silver nanparticle with the parameters $\mathrm{L} 1=0.33, \mathrm{~L} 2=0.37, \mathrm{~L} 3=0.39, \varepsilon_{h}=2.25, P=0.99, \gamma=1.15 \times 10^{-2}, \varepsilon_{\infty}=4.5$.The induced optical bistability in the plane $\left(z, \chi\left|E_{h}\right|^{2}\right)$

\subsection{Bistability Domain in Ellipsoidal Particles with Nonlinear Metal/Dielectric Composite Core}

The bistability domain in the plane $\left(z, \chi\left|E_{h}\right|^{2}\right)$ can be specified from an analysis of the roots of the cubic equation (35). We have two ways of finding the root location of this cubic equation. The solution of equation (35) is:

$$
\boldsymbol{Y}=\frac{-2}{9}\left(\left(\mathbf{b}^{2}-3 a c\right)\left(-\mathbf{b} \pm \frac{\sqrt{\mathbf{b}^{2}-3 a c}}{3 a}\right)+\frac{\mathbf{b c}}{2}\right)
$$

As we see from Fig 8, taken the depolarization for spherical shape $L=1 / 3$, the bistability domain have M-like shape where it start increasing from point ( 0.75 to 3$)$ for $\chi\left|E_{h}\right|^{2}$ and (0.33 to 0.35) and decreased from (0.36 t0 $0.4)$ for $z$. Between a point of z (0.34 to 0.36$)$ we have the bistability domain in which we find a particle at a particular point.

From fig 9 we understand for $\left(z, \chi\left|E_{h}\right|^{2}\right)$ frequency and intensity there is no change in bistability domain of silver nanparticle. The combination of three nanoparticles shape display similar behavior and characteristics except they are different for different depolarization factor. The most interesting feature of these graphs is the appearance of two peaks of the enhancement factor at two $(\mathrm{z}=0.3,0.4)$; different frequencies. The positions and values of these maxima strongly depend on volume fraction $\mathrm{P}$ (for fixed other parameters). For $\mathrm{P}=0.99$, it becomes more important and with further increase in $\mathrm{P}$, three of them become higher and move closer to each other along positive $\mathrm{X}$-axis, whereas further increase in $\mathrm{P}$, three of them become lower and move parallel to each other and decrease to zero.

Generally, we conclude that the metallic/dielectric composite core for ellipsoidal nanoparticle shows induced optical bistability and bistability domain have the same result as spherical nanoparticle metallic/dielectric composite core.

\section{Conclusions}

From the study, we observed that for incident radiation
(EMW) the dielectric function in ellipsoidal nanoparticle have a great effect on enhancement factor and it have two maximum peaks for depolarization factor $\mathrm{L}$ and dimensionless frequency $z$. The fundamental point we discussed is optical bistability, in which the intensity of output light can take two distinct stable values for a given input incident radiation, creating optical bistable for real states output and one absorbed for imaginary part. The degree of optical nonlinearity in a material depends upon the strength of the optical field, and varies in different materials. The bistability domain for spherical and ellipsoidal nanoparticle show the output result the same for input local electric field but they are not overlap one another, and shift their place to the left for oblate and to right for prolate in compared to spherical.. It is shown that the enhancement factor of the local electric field in metal spherical or ellipsoidal nanoparticles with dielectric cores imbedded in a dielectric matrix have two maxima on two resonant frequencies. The second maximum for the inclusions with large dielectric cores covered by a thin metal shell is comparatively small. With increasing in a metal fraction in the inclusion, enhancement factor grows. The optical bistability domain is observed in the range of the depolarization factor of $L=0.34$ and $L=0.4$, which has one domain for ellipsoidal composite case.

\section{Acknowledgements}

The authors are grateful to Jimma University for financial support and the authors of the referred materials.

\section{REFERENCES}

[1] Abdulrhman Al-Warthan, Kholoud M. M. Abou El-Nour, Alaa Eftaiha and Reda A. A. Ammar B. Synthesis and applications of silver nanoparticles. Arabian Journal of chemistry, page 3, 135-140, (2010). 
[2] A. Stalmashonak, et. al. Optical Properties of Nanocomposites Containing Metal Nanoparticles (2013).

[3] A. S. B. Shambra, et.al. Bistability in an Acousto-Optic Tunable Filter Operating with Short Optical Pulses. The Industrial Physicists, received December 30th, 2011; revised February 5th, 2012; accepted February 15th, (2012).

[4] Ineke Malsch. Biomedical Applications of Nanotechnology. The Industrial Physicists, 8(3), (2002).

[5] F. Bohren and D. R .Huffman, Absorption and scattering of light by small particles, Wiley, New York, 1983.

[6] V. N. Mal'nev S. Shewamare, O. A. Buryi. Induced Optical Bistability in Small Metal and Metal Coated Particles with Nonlinear Dielectric functions. Ukr. J, 56(4): 657 -660, 2012.

[7] R. S. Bennink Y. H. Yoon and R. W. Boyd. Accessing the optical nonlinearity of metals with metal-dielectric photonic bandgap structures. Teubner, Leipzing, 24: 1416 - 1418, 1999.

[8] K.M. Leung, et al. Optical bistability of an aqueos aerosol particle detected through light scattering: theory and experiment. Apps. Optics, 29:3473, 1990

[9] Ohad Levy, D.J, Bergman. Theory of optical bistability in weakly nonlinear composite medium. 49:129-134, 1994.

[10] S. Arnold, T. R. Keeffe, K. M. Leung, L. M. Floan, T. Scalese and A. Pluchino. Optical bistability of an aqueous aerosol particle detected through light scattering: theory and experiment. Appl. Optics, 29:3473, 1990.

[11] R. Nathanshon Levy and D. J. Bergman. Electrical resonance and optical bistability in periodic composite materials. J. Appl. Phys, 77:4263-4273, 1995.

[12] Ohad Levy and D.J. Bergman. Harmonic generation, induced nonlinearity, and optical bistability in nonlinear composites. Phys. stat. sol. (b), 52(3), 1995.

[13] B. R. Master. Handbook of Biomedical Nonlinear Optical Microscopy. Press, 1995.

[14] C. Rulliere. Femtosecond Laser Pulses: Principles and Experiments. Springer, 2nd edition, 2004.

[15] L.S. Tan Q. Zheng, G.S He and P.N. Prasad. Multiphoton absorbing materials: Molecular designs, characterizations, and applications. Chemical Review, 108(2008):1245-1330.
[16] G.P. Agrawal. Nonlinear Fiber Optics, Optics and Photonics Series. Academic Press, 2003.

[17] G.P. Agrawal. Lightwave technology components and devices. Jhon Wiley and Sons, New Jersey, 2004.

[18] H. Nihei and A. Okamoto. Photonic crystal systems for high-speed optical memory device on an atomic scale. Pro.SPIE, 4416:470-473, 2001.

[19] S. Omi H. Tanji T. Tokzaki A. Nakamura S. Kaneko, K. Uchida and Y. Asaharaaj. Subpicosecond time response of third - order optical nonlinearity of small copper particles in glass. Applied physics Letters, 65: 941 - 943, 1994.

[20] V. M. Shalaev. Nonlinear Optics of Random Media. Springer - Verlag, 2000.

[21] A. P. Gonzalez - Marcos. Optical bistable devices as sensing elements, Unmanned/Unattended sensors and sensors Networks. Edited by Edward M. Carapezza, proceedings of SPIE, 5611 (SPIE, Bellingham, WA), 2004.

[22] D. J. Hagan G. Assanto, Z. Wang and E. W. Vanstryland. All - optical modulation via nonlinear cascading in type II second - harmonic generation. Appl. phys Lett, pages 67 (15) 2120 $2122,1995$.

[23] D. A. Mazurenko, and R. Kerst, et.al. Ultrafast optical switching in three - dimensional photonic crystals, Phys. Rev. Lett, (91 (21) 2139 03): 2003.

[24] G. Priem, P. Dumon, W. Bogaerts, D. Van Thought, G. Morthier, and R. Baets, Ultrafast optical switching in three dimensional Photonic crystals. Opt. Express, (23) (13): $9623-9628,2005$.

[25] Y. Wang, G. X. Li, H. L. Tam, K. W. Cheah, and S. N. Zhu. Optical bistability and multistability in one dimensional periodic metal-dielectric photonic crystal, Appl. Phys. Lett, 21(211109), 92, 2008.

[26] F. Yanik, S. H. Fan, M. Soljacic, and J. D. Joannopoulos. All-optical transistor action with bistable switching in a photonic crystal cross-waveguide geometry, Phys. Rev. Lett., 28(24), 2506-2508, 2003.

[27] F. Yanik, S. H. Fan, and M. Soljacic. High-contrast all-optical bistable switching in photonic crystal microcavities, Appl. Phys. Lett., 83 (14), 2739-2741, 2003. 